This is the final peer-reviewed accepted manuscript of:

Boanini E*, Torricelli P, Bonvicini F, Cassani MC, Fini M, Gentilomi GA, Bigi A. A new multifunctionalized material against multi-drug resistant bacteria and abnormal osteoclast activity. European Journal of Pharmaceutics and Biopharmaceutics 2018; 127:120-129.

The final published version is available online at: $h$ ttp://dx.doi.org/10.1016/i.ejpb.2018.02.018

Rights / License:

The terms and conditions for the reuse of this version of the manuscript are specified in the publishing policy. For all terms of use and more information see the publisher's website.

This item was downloaded from IRIS Università di Bologna (https://cris.unibo.it/)

When citing, please refer to the published version. 


\section{A new multifunctionalized material against multi-drug resistant bacteria and abnormal osteoclast activity.}

Elisa Boanini ${ }^{\text {a, } * \text {, Paola Torricelli }}{ }^{\text {b }}$, Francesca Bonvicini ${ }^{c}, *$, Maria Cristina Cassani ${ }^{\mathrm{d}}$, Milena Fini ${ }^{\mathrm{b}}$, Giovanna Angela Gentilomi ${ }^{\mathrm{c}}$ and Adriana Bigi ${ }^{\mathrm{a}}$

\section{Affiliations:}

a Department of Chemistry "Giacomo Ciamician", University of Bologna, via Selmi 2, 40126 Bologna, Italy

b Laboratory of Preclinical and Surgical Studies, Codivilla-Putti Research Institute, Rizzoli Orthopaedic Institute, via di Barbiano 1/10, 40136 Bologna, Italy

${ }^{\mathrm{c}}$ Department of Pharmacy and Biotechnology, University of Bologna, via Massarenti 9, 40138 Bologna, Italy

${ }^{\mathrm{d}}$ Department of Industrial Chemistry "Toso Montanari", University of Bologna, viale del Risorgimento 4, 40136 Bologna, Italy

\section{Corresponding Authors:}

*Elisa Boanini; e-mail address: elisa.boanini@unibo.it

*Francesca Bonvicini; e-mail address: francesca.bonvicini4@unibo.it 


\begin{abstract}
The development of new biomaterials able to favor bone formation and to inhibit bone abnormal resorption is mandatory to face the increasing number of age-related musculoskeletal disorders. Moreover, the increasing antibiotic resistance of clinically important bacteria, which is among the main causes of implant failure, requires new antimicrobial systems. In this study, we prepared multifunctional materials consisting of hydroxyapatite-zoledronate composite crystals decorated with Ag Nanoparticles (AgNPs). Zoledronate, a potent bisphosphonate widely applied for the treatment of pathologies associated to abnormal bone loss, was incorporated into hydroxyapatite up to about $8 \mathrm{wt} \%$. Loading of poly(ethylenimine) - stabilized AgNPs onto the crystals was promoted by zoledronate functionalization and provoked a significant variation of the values of zeta potential. The results of in vitro tests demonstrate that the multifunctional materials combine the beneficial actions of zoledronate and AgNPs. In fact, they improve osteoblast differentiation and activity, whereas they inhibit osteoclastogenesis and osteoclast differentiation, and significantly hinder the growth of multi-drug resistant Gram positive and Gram negative bacteria. As a consequence, they can be exploited both as antiresorptive agents and as antimicrobial materials able to prevent the development of bone-associated infections.
\end{abstract}

Keywords: bisphosphonate; zoledronate; silver nanoparticles; hydroxyapatite; multidrug resistant bacteria; osteoblast / osteoclast co-cultures; osteoporosis.

This item was downloaded from IRIS Università di Bologna (https://cris.unibo.it/)

When citing, please refer to the published version. 


\section{Introduction}

Thanks to its similarity to the inorganic phase of bone, hydroxyapatite (HA) is the most employed calcium phosphate for the preparation of biomaterials for the repair/substitution of the hard tissues of vertebrates. The good biocompatibility and bioactivity of HA can be enhanced and improved through functionalization with ions and molecules capable to promote bone growth and to inhibit bone abnormal resorption, as well as to prevent infections and/or adverse immune reactions [1-3]. In particular, functionalization with bisphosphonates (BPs) was shown to provide composite materials able to hinder abnormal osteoclast mediated resorption [4-6]. BPs are widely employed for the treatment of pathologies characterized by excessive bone turnover [7]. Moreover, nitrogen containing bisphosphonates (NBPs), which inhibit farnesyl pyrophosphate synthase (FPP) enzyme within the mevalonate pathway in osteoclasts, have both direct and indirect antitumor activities [8]. Zoledronate (ZOL), which has been reported to exhibit a high binding activity to synthetic HA [9], is one of the most potent antiresorptive NBPs [10].

On the other hand, several undesirable side effects, including osteonecrosis of the jaw and atypical subtrochanteric fractures, have been associated to the prolonged systemic administration of these drugs $[11,12]$. In order to avoid/reduce these drawbacks, a number of different materials, including calcium phosphates, have been proposed as delivery systems for N-BPs local administration at specific bone sites [13,14]. In particular, we have previously demonstrated that zoledronate functionalized HA (HAZOL) promotes in vitro osteoblast viability and activity, whereas it inhibits osteoclast proliferation and differentiation $[5,15,16]$. Herein we used HAZOL as support for silver nanoparticles 
(AgNPs) with the aim to get composite materials able to display both antiresorptive and antimicrobial properties.

Bacterial colonization of implants and medical devices is a big problem since clinically important bacteria exhibit increasing antibiotic resistance [3]. Silver nanoparticles display strong antimicrobial properties against a wide number of bacteria [17-20]. AgNPs are less toxic than silver ions, which can damage bacterial DNA, proteins, enzymes and cell walls [21-23]. Toxicity seems to depend on several nanoparticles properties, such as surface area, size and shape, surface charge, particle purity [24]. Anyhow, the concentrations for antimicrobial activity were reported to be much less than that capable to provoke cytotoxicity [25]. Clinical results on orthopedic applications of AgNPs, although still limited, are encouraging and supported by a number of in vitro and in vivo studies [26,27]. Silver doped/substituted HA has been the object of a number of studies [28-31], whereas a few investigations on the antibacterial activity of AgNPs supported on HA particles have been reported [32-36]. In this study we loaded different amounts of polyethylenimine (PEI) stabilized AgNPs onto HAZOL, as well as onto pure HA as control. To this purpose, we used low molecular weight PEI, in order to avoid the cytotoxicity problems that have been reported for the cationic polymer at high molecular weight [37]. Bone cells response was investigated using an osteoblast-osteoclast coculture system. In particular, the study was performed using osteoclast (OC) derived from osteoporotic subjects in order to focus the attention on the action of ZOL on OC with abnormal activity. In fact osteoporotic OC show a different behavior when compared to normal ones, causing an impairment of the balance between bone resorption and formation [38-40]. The antibacterial properties of the composite materials were tested in vitro against a panel of Gram positive and Gram negative reference bacterial strains 
including pathogens frequently involved with implant-associated infections and against antibiotic-resistant clinical isolates recovered from patients with chronic bone or prosthetic joint infections.

\section{Materials and Methods}

\subsection{Synthesis and Characterization of Materials}

The synthesis of hydroxyapatite (HA) was carried out in $\mathrm{N}_{2}$ atmosphere using $50 \mathrm{~mL}$ of $1.08 \mathrm{M} \mathrm{Ca}\left(\mathrm{NO}_{3}\right)_{2} \cdot 4 \mathrm{H}_{2} \mathrm{O}$ at $\mathrm{pH}$ adjusted to 10 with $\mathrm{NH}_{4} \mathrm{OH}$. The solution was heated at $90{ }^{\circ} \mathrm{C}$ and $50 \mathrm{~mL}$ of $0.65 \mathrm{M}\left(\mathrm{NH}_{4}\right)_{2} \mathrm{HPO}_{4}, \mathrm{pH} 10$ adjusted with $\mathrm{NH}_{4} \mathrm{OH}$, was added dropwise under stirring. After 5 hours at $90{ }^{\circ} \mathrm{C}$ under stirring, precipitate was centrifuged at $10,000 \mathrm{rpm}$ and repeatedly washed with $\mathrm{CO}_{2}$-free distilled water. The product was dried at $37{ }^{\circ} \mathrm{C}$ overnight, then mildly ground in an agate mortar and sieved $(\mathrm{d}=56 \mu \mathrm{m})$. Zoledronate-containing hydroxyapatites were obtained by following the same procedure, and by adding disodium zoledronate tetrahydrate (Chemos $\mathrm{GmbH}$ ) to the phosphate solution [5]. Zoledronate concentrations calculated on final volume were 7 and $14 \mathrm{mM}$, thereby samples were labelled ZOL7 and ZOL14, respectively.

Bisphosphonate content was determined spectrophotometrically via complex formation with Fe(III) ions using a Varian Cary50Bio instrument $(\lambda=290 \mathrm{~nm})[41]$.

PEI-stabilized Ag nanoparticles (AgNPs) were prepared by heating $100 \mathrm{~mL}$ of $10 \mathrm{mM}$ $\mathrm{AgNO}_{3}$. Once at the boiling point, $0.7 \mathrm{~mL}$ of $10 \%(\mathrm{w} / \mathrm{w})$ polyethylenimine $(\mathrm{MW} \approx 2000)$ solution were quickly added. Resulting boiling solution gradually turned brown and after 4 min it was allowed to cool at room temperature. Finally, to account for water evaporation, the volume was adjusted to $100 \mathrm{~mL}$.

This item was downloaded from IRIS Università di Bologna (https://cris.unibo.it/)

When citing, please refer to the published version. 
HA-AgNPs, ZOL7-AgNPs and ZOL14-AgNPs aggregates were prepared after incubation of $0.500 \mathrm{~g}$ of HA or ZOL7 or ZOL14 powders in freshly prepared AgNPs colloidal solution, just cooled at room temperature. The resulting suspension was stirred for $1 \mathrm{~h}$. After filtration on a Buchner funnel, the solid was thoroughly washed with water and dried at $37^{\circ} \mathrm{C}$. Different volumes of AgNPs solution were tested, namely 5, 10, 20, 30, 40, and $50 \mathrm{~mL}$. Accordingly, samples were labelled HA-5, HA-10, HA-20, etc.; ZOL7-5, ZOL7-10, ZOL7-20, etc.; and ZOL14-5, ZOL14-10, ZOL14-20, etc.

The same procedure was utilized to prepare some further samples containing PEI but no AgNPs, as control materials for cytotoxicity and antibacterial tests. In detail, the samples were prepared through incubation of $0.500 \mathrm{~g}$ of HA or ZOL14 in 5, 20 or $50 \mathrm{~mL}$ of a solution obtained by boiling $100 \mathrm{ml}$ of an aqueous solution containing $0.7 \mathrm{~mL}$ of $10 \%$ (w/w) PEI for 4 minutes and then cooling at room temperature. The resulting suspensions were stirred for $1 \mathrm{~h}$. After filtration on a Buchner funnel, the solids were thoroughly washed with water and dried at $37{ }^{\circ} \mathrm{C}$. The samples were labeled HA-20P, HA-50P, ZOL14-5P and ZOL14-50P.

X-ray diffraction (XRD) analysis was carried out by means of a PANalytical X'Pert PRO powder diffractometer equipped with a fast $\mathrm{X}^{\prime}$ Celerator detector. Ni-filtered $\mathrm{CuK} \alpha$ radiation was used $(40 \mathrm{~mA}, 40 \mathrm{kV})$. For phase identification the $2 \theta$ range was investigated from 10 to $602 \theta$ degrees with a step size of $0.1^{\circ}$ and time/step of $100 \mathrm{~s}$.

The amount of silver present on the different samples was determined with flame atomic absorption spectroscopy (AAS, Thermo Scientific) in air-acetylene flame with a wavelength of $328.1 \mathrm{~nm}$ and a spectral band-width of $0.5 \mathrm{~nm}$. The analyses were conducted dissolving ca. $8 \mathrm{mg}$ of previously grinded solid samples (weighted with a 
Mettler Toledo AT 21 Comparator balance), in $25 \mathrm{ml}$ of a $0.5 \mathrm{M} \mathrm{HNO}_{3}$ aqueous solution. The calibration line was made with 5 calibration standards $(2,4,6,8,10 \mathrm{ppm})$, prepared by dilution to $50 \mathrm{~mL}$ of a 100 ppm silver standard for AAS in $0.5 \mathrm{M} \mathrm{HNO}_{3}$ (Merck). For Transmission Electron Microscopy (TEM) investigations, a drop of sonicated sample suspension in ethanol was transferred onto holey carbon foils supported on conventional copper microgrids. A Philips CM100 transmission electron microscope, operating at 80 $\mathrm{kV}$ was used.

Zeta potential was measured using Electrophoretic Light Scattering (ZetasizerNano; Malvern Instruments). $5 \mathrm{mg}$ of powder sample was suspended in $50 \mathrm{~mL}$ of MilliQ water and sonicated for 2 minutes before zeta potential measurement. Each analysis was performed in triplicate.

The specific surface area was measured using a Carlo Erba Sorpty 1750 BET analyser using constant volume $\mathrm{N}_{2}$ absorption with desorption at $80{ }^{\circ} \mathrm{C}$.

For infrared (FT-IR) adsorption analysis, $1 \mathrm{mg}$ of the powdered samples was carefully mixed with $200 \mathrm{mg}$ of $\mathrm{KBr}$ (infrared grade) and pelletized under a pressure of 10 tons for 1 min. The pellets were analyzed using a Bruker ALPHA FT-IR spectrophotometer to collect 32 scans in the range $4000-400 \mathrm{~cm}^{-1}$ at a resolution of $4 \mathrm{~cm}^{-1}$.

In vitro tests were performed on disk-shaped samples $(\emptyset=6.0 \mathrm{~mm})$. Each disk was prepared by pressing $40 \mathrm{mg}$ of powder into cylindrical moulds by using a standard evacuable pellet die (Hellma). Disk shaped samples for antibacterial activity, cytotoxicity and cell co-culture were sterilized using gamma rays.

Release of Silver from disk-shaped samples was measured in the medium used for cell culture differentiation (see section 2.4). The supernatants were removed from the wells at increasing times up to 21 days and Ag content was analyzed using flame atomic 
absorption spectroscopy (AAS, Thermo Scientific) in air-acetylene flame with a wavelength of $328.1 \mathrm{~nm}$ and a spectral band-width of $0.5 \mathrm{~nm}$. Results from this analysis represent the mean value of three different determinations.

\subsection{Bacterial strains and Kirby-Bauer disk diffusion method}

The in vitro antibacterial activity of the composite materials (HA-5, HA-20, HAZOL7-5, HAZOL7-20, HAZOL14-5, HAZOL14-20) was preliminary evaluated against a panel of Gram positive and Gram negative reference bacterial strains including Staphylococcus aureus (ATCC 25923), Staphylococcus epidermidis (ATCC 12228), Enterococcus faecalis (ATCC 29212), Escherichia coli (ATCC 25922), Klebsiella pneumoniae (ATCC 9591), Pseudomonas aeruginosa (ATCC 27853). Subsequently, having defined both the overall spectrum of antibacterial activity and the cytotoxic profile of the tested samples, HA-20 and HAZOL14-5, which contain almost the same amount of AgNPs (0.8-0.9 wt $\%)$, were assayed towards 10 clinical isolates recovered from patients with chronic bone or prosthetic joint infections. Strains included 5 S. epidermidis of which 3 methicillinresistant (MRSE) and 5 P. aeruginosa of which 2 multi-drug resistant (MDR); they were isolated on BD Columbia Agar with 5\% sheep blood (Becton Dickinson, GmbH, Germany) and confirmed by MALDI-TOF MS (Bruker Daltonik, GmbH, Germany) [42]. Their antibiotic susceptibility was determined by using the Vitek2 semi-automated system (bioMerieux, France) and interpreted following the EUCAST susceptibility testing guidelines [43].

The Kirby-Bauer disk diffusion method $(\mathrm{KB})$ was performed to determine the diameter of the inhibition zone (corresponding to the bacterial-free zone on an agar plate) surrounding the disk-shaped samples $(\varnothing=6.0 \mathrm{~mm})$, and was carried out following the requirements of the CLSI (2015) [43,44]. Briefly, the surface of Mueller-Hinton agar

This item was downloaded from IRIS Università di Bologna (https://cris.unibo.it/)

When citing, please refer to the published version. 
plate (MHA) (Sigma-Aldrich) was inoculated with the bacterial suspension at 0.5 McFarland, prepared in sterile $0.9 \%$ saline solution. Gamma rays sterilized disks were placed on the agar plates and incubated at $37^{\circ} \mathrm{C}$ for 24 hours. Disks containing gentamicin $(\mathrm{GMN} 10 \mu \mathrm{g})$ and/or imipenem (IPM $10 \mu \mathrm{g})($ Oxoid SpA, Italy) were included as positive controls while pure HA, HAZOL and composite samples containing PEI but not AgNPs were used as negative controls. The content of antibiotics, gentamicin and imipenem, is set at $10 \mu \mathrm{g}$ according to the procedures established by a number of committees, such as The Clinical Laboratory Standards Institute (CLSI) in the US, or The European Committee on Antimicrobial Susceptibility Testing (EUCAST) in Europe.

After incubation, the diameter of the growth inhibition was measured and expressed in millimeters (mm). All experiments were performed on duplicate in different days.

\subsubsection{Bacterial strains and MIC determination}

The in vitro antibacterial activity of $\mathrm{Ag}^{+}$ions was evaluated for the reference bacterial panel by a broth microdilution method using a 96 -well plate $[43,44]$. Briefly, the bacterial suspension, prepared at 0.5 McFarland, was diluted 1:200 in Mueller-Hinton (MH) broth (Sigma-Aldrich) or in Brain Heart Infusion (BHI) broth (Biolife), and incubated with serial two-fold dilutions of $\mathrm{Ag}^{+}$ions from $50 \mu \mathrm{g} / \mathrm{mL}$ to $0.05 \mu \mathrm{g} / \mathrm{mL}$, and of gentamicin (Sigma-Aldrich) from $5 \mu \mathrm{g} / \mathrm{mL}$ to $0.005 \mu \mathrm{g} / \mathrm{mL}$, as reference drug. Each strain and all controls (bacterial cells grown in regular medium, and dilutions of $\mathrm{Ag}^{+}$ions and gentamicin to check the background turbidity of reagents and the sterility of the procedures) were tested in triplicate, and in two independent experiments. The microplate was incubated at $37^{\circ} \mathrm{C}$ for $24 \mathrm{~h}$, and subsequently the Optical Density at $630 \mathrm{~nm}$ was measured by the Multiskan Ascent microplate reader (Thermo Fisher Scientific Inc., Waltham, USA). Percentage values of samples at the different experimental conditions 
were determined as relative to the positive growth control and $\mathrm{MIC}_{90}$ values (Minimum Inhibitory Concentration), corresponding to the lowest $\mathrm{Ag}^{+}$concentration giving rise to an inhibition of bacterial growth $\geq 90 \%$ were obtained by the interpolation on the doseresponse curves. Statistical analysis was carried out by nonlinear regression method using GraphPad Prism version 5.00 for Windows (GraphPad Software, San Diego California, USA).

\subsection{In vitro cytotoxicity}

MG63 human osteoblast cell line was expanded in Dulbecco's Modified Eagle Medium (DMEM, Sigma, UK) supplemented with 10\% FCS, and antibiotics (100 U/ml penicillin, $100 \mu \mathrm{g} / \mathrm{ml}$ streptomycin). After trypsinization, cells number and viability were checked by trypan blue dye exclusion test. Cells were plated at a density of $2 \times 10^{4}$ cells $/ \mathrm{mL}$ in $24-$ well plates onto and around sterile samples of the different materials. Cells were also seeded in wells for negative (CTR-, DMEM only) and positive (CTR+, DMEM $+0.05 \%$ phenol solution) controls for cytotoxicity tests, (according to UNI EN ISO 10993-5, Biological evaluation of medical devices - Part 5. Tests for in vitro cytotoxicity). Plates were cultured in standard conditions, at $37 \pm 0.5^{\circ} \mathrm{C}$ with $95 \%$ humidity and $5 \% \pm 0.2 \mathrm{CO}_{2}$ for $72 \mathrm{~h}$.

The evaluation of cytotoxicity was performed by measuring cell viability and lactate dehydrogenase enzyme (LDH) release. Cell proliferation and viability was assessed by WST1 (WST1, Roche Diagnostics GmbH, Manheim, Germany) colorimetric reagent test. The assay is based on the reduction of tetrazolium salt to a soluble formazan salt by a reductase of the mitochondrial respiratory chain, active only in viable cells. $100 \mu \mathrm{l}$ of WST1 solution and $900 \mu \mathrm{l}$ of medium (final dilution: 1:10) were added to the cell 
monolayer, and the multi-well plates were incubated at $37^{\circ} \mathrm{C}$ for a further $4 \mathrm{~h}$. Supernatants were quantified spectrophotometrically at $450 \mathrm{~nm}$ with a reference wavelength of $625 \mathrm{~nm}$. Results of WST1 are reported as optical density (OD) and directly correlate with the cell number.

At the end of experimental time the supernatant was collected from all wells and centrifuged to remove particulates, if any, to detect Lactate Dehydrogenase (LDH, enzyme-kinetic test, Roche Diagnostics $\mathrm{GmbH}$ ) release: $100 \mu \mathrm{l}$ of reagent were added to $100 \mu \mathrm{l}$ of cell supernatant in a 96-wells plate; after $30 \mathrm{~min}$ of incubation at room $\mathrm{T}$ in the dark, samples were evaluated by spectrophotometer at 490/655 $\mathrm{nm}$.

A qualitative analysis for cell morphology was performed by Neutral Red (NR) vital staining. A $0.033 \%$ solution of NR staining (Sigma, UK) in culture medium was added to two wells for each group at the end of experimental times, for further $90 \mathrm{~min}$. Cultures were examined by light microscopy for the evaluation of cell morphology.

\subsection{In vitro co-cultures}

Human primary monocytes (OC) and human osteoblast-like cells MG63 (OB, Istituto Zooprofilattico Sperimentale IZSBS, Brescia, Italy), were used for the co-culture model. Peripheral human blood was obtained from osteoporotic adult volunteers (Rizzoli Orthopedic Institute Ethic Comittee approval n.33154, 12/09), who presented a recent diagnosis by densitometry and with no therapy at the time. Density gradient centrifugation was used to separate the mononuclear cells from the other elements of blood. Briefly, a volume of peripheral blood was diluted 1:1 with pre-warmed PBS, carefully layered on an equal volume of Histopaque1077 (Sigma, MO, USA), and was centrifuged at $600 \mathrm{~g}$ at room temperature for $30 \mathrm{~min}$. After centrifugation, the

This item was downloaded from IRIS Università di Bologna (https://cris.unibo.it/)

When citing, please refer to the published version. 
mononuclear cells accumulated at the interface between PBS and Histopaque were collected and washed twice in PBS. Pellet was resuspended in $1 \mathrm{ml}$ of culture medium (DMEM, basal medium $+10 \%$ FBS, $1 \%$ antibiotics; Sigma, UK); cell number and viability were checked with trypan blue (Sigma, UK) dye exclusion test for experiment. Monocytes at a concentration of $4 \times 10^{5}$ cells/well were differentiated in the bottom of 24 well plates containing samples of HA (as reference), HA-20, ZOL14, ZOL14-5. They were incubated at $37^{\circ} \mathrm{C}$ in a humidified $95 \%$ air $/ 5 \% \mathrm{CO}_{2}$ atmosphere (standard condition). After 24 hours the non-adherent monocytes were washed off to dispose the culture of contaminating lymphocytes, so that only the adherent monocytes were used for culture. DMEM additioned with macrophage colony-stimulating factor (MCSF, 25ng/ml; ) and receptor activator for $\mathrm{\kappa B}$ factor ligand (RANKL, 30ng/ml; ) was used for OC differentiation for 1 week.

OB were expanded in DMEM supplemented with 10\% FCS, $1 \%$ antibiotics $(100 \mathrm{U} / \mathrm{ml}$ penicillin, $100 \mu \mathrm{g} / \mathrm{ml}$ streptomycin), $\beta$-Glycerophosphate $\left(10^{-4} \mathrm{M}\right)$ and Ascorbic acid (50 $\mu \mathrm{g} / \mathrm{ml}$; Sigma, UK). After 1 week, OB were counted, plated on material samples at a concentration of $3 \times 10^{4}$ cells/well and co-cultured with OC. It was set up a control coculture (CTR) without material by seeding OB in transwell inserts $0.4-\mu \mathrm{m}$ pore size (Millipore, Ireland) and OC in the bottom of the well (Figure S1).

Both cell types were cultured in proportional osteoblast:osteoclast differentiation medium up to 14 days.

\subsubsection{Cell viability}

OC and OB proliferation and viability was evaluated by WST1 colorimetric reagent test (Roche Diagnostics GmbH, Manheim, Germany) at the end of experimental time, as described above. To allow assessment of proliferation of each cell type separately, co- 
cultures were disassembled, and materials, as well as transwells with OB, were transferred in empty wells.

\subsubsection{Osteoclastogenesis}

TRAP-staining was performed to assess osteoclast differentiation according to manufacturer's instructions (SIGMA, Buchs, Switzerland). The positive cells developed red colour of different intensity. The number of TRAP-positive multinucleated cells (three or more nuclei each cell) was counted under the microscope by a semiautomatic software (NIS-Elements AR 4.30.01) and results are given as percentage of CTR OC, considered as $100 \%$.

\subsubsection{Osteoclast and osteoblast activity}

At the end of experimental time, after 14 days of culture, the supernatant was collected from all wells and centrifuged to remove particulates, if any. Aliquots were dispensed in Eppendorf tubes for storage at $-70^{\circ} \mathrm{C}$ and assayed with the following immunoenzymatic kits: Alkaline Phosphatase (ALP, CloudeClone Corp., Wuhan, China), Osteocalcin (OSTC, e-Bioscience, Bender MedSystems, Vienna, A), Osteoprotegerin (OPG, Boster Biological Technology, Ca, USA), Receptor Activator for Nuclear factor KB Ligand (RANKL, Boster), Interleukin-6 (IL-6, CloudeClone Corp.), and Tumor necrosis factor $\alpha$ (TNF- $\alpha$, Boster). Co-culture medium additioned with RANKL was evaluated and subtracted from RANKL samples values. Data are normalized by WST1 values.

\subsubsection{Osteoblast morphology}

At the end of the experiment, samples of each material were prepared for SEM: osteoblasts grown on the materials were fixed in $2.5 \%$ glutaraldehyde, in phosphate buffer $0.01 \mathrm{M}(\mathrm{pH} 7.4)$ for $1 \mathrm{~h}$, and then dehydrated in a graded ethanol series. After a passage in hexamethyldisilazane, the samples were air dried. The samples were sputter-coated 
with Pd before examination with a Hitachi S-2400 instrument operating at $15 \mathrm{kV}$.

\subsection{Statistical Analysis}

Statistical evaluation of cytotoxicity and co-cultures data was performed using the software package SPSS/PC ${ }^{+}$Statistics ${ }^{\mathrm{TM}} 23$ (SPSS Inc., Chicago, IL USA). The results presented are the mean of three cytotoxicity tests and six co-cultures independent values. Data are reported as mean \pm standard deviations (SD) at a significance level of $p<0.05$. A one-way ANOVA was done for comparison among groups. Finally, a post hoc multiple comparison tests was performed to detect significant differences among groups, and Pearson test was performed to detect correlation between data.

\section{Results and Discussion}

\subsection{Chemical and structural characterization}

All the samples used as substrates for AgNPs loading are constituted of hydroxyapatite as unique crystalline phase. Functionalization of hydroxyapatite with zoledronate provokes a decrease of crystallinity, as shown by the broadening of the XRD peaks and of the FT-IR absorption bands on increasing ZOL concentration in the synthesis solution (Figure S2), in agreement with previous results [5]. Moreover, TEM images display a reduction of the mean dimensions of the crystals on passing from HA to ZOL7, to ZOL14 (Figure S3), coherently with the increase of the surface area (Table S1). The amount of bisphosphonate in the different samples increases with its concentration in solution up to about $8.6 \mathrm{wt} \%$. The presence of ZOL induces a shift of the zeta potential towards more negative values: the zeta potential of $\mathrm{HA}$ is $-11.5 \mathrm{mV}$ and decreases down to $-26.4 \mathrm{mV}$ for ZOL14 (Table S1). The negatively charged surfaces should promote interaction with

This item was downloaded from IRIS Università di Bologna (https://cris.unibo.it/)

When citing, please refer to the published version. 
AgNPs, which exhibit a positive superficial charge due to the presence of polyethylenimine [36]. As a matter of fact, the zeta potential of PEI-stabilized AgNPs obtained through dynamic light scattering measurement is $+37 \mathrm{mV}$ [36].

The results of chemical analysis show that the amount of AgNPs associated to HA reaches a maximum of about $1 \mathrm{wt} \%$, whereas the amounts of AgNPs onto ZOL7 and ZOL14 increase with the volume of colloidal suspension up to 1.8 and $2.4 \mathrm{wt} \%$, respectively (Table1). Figure 1 reports the XRD patterns of the different samples obtained after interaction of the apatitic materials with $50 \mathrm{~mL}$ of AgNPs suspension. The patterns show the presence of a number of diffraction reflections characteristic of hydroxyapatite (PDF 9-432). Moreover, a reflection at about $38.1^{\circ} 2 \theta$, corresponding to the most intense peak of $\mathrm{Ag}$, indicates the presence of metallic silver (PDF 4-783).

The relative intensity of Ag reflection, which increases in the order HA-50, ZOL7-50, ZOL14-50, in agreement with the results of chemical analysis. In the same way, TEM images reported in Figure 2 show that the number of AgNPs appreciable onto ZOL14 is greater than that detectable onto ZOL7 and HA. AgNPs size does not vary in the different samples, and assumes a mean value of $20 \mathrm{~nm}$. A typical size histogram is reported in Figure S4.

The adsorption of the nanoparticles onto the apatitic samples provokes an increase of the zeta potential, which assumes values ranging from $+7.3 \mathrm{mV}$ to $+5.3 \mathrm{mV}$ on passing from HA to ZOL14 samples, without significant variation as a function of silver content. Even relatively small amounts of adsorbed AgNPs cause significant increase of the values of zeta potential, most likely because of relatively high presence of the polycationic PEI on the surface of the nanoparticles.

This item was downloaded from IRIS Università di Bologna (https://cris.unibo.it/) 
Ag release from HA-20 and ZOL14-5 during co-culture cell tests was measured in cell culture medium as a function of time up to 21 days. The results, reported in Figure 3 , show an initial burst release followed by a steady profile for both samples, with slightly smaller values for HA-20. The cumulative release reaches values of about 65-70 $\mu \mathrm{g}$ (16$18 \mathrm{wt} \%$ ) in the first 2 weeks, after which it assumes almost constant values of $72-75 \mu \mathrm{g}$ $(18-19 \mathrm{wt} \%)$. The similar release kinetics of the two different samples is not surprising since they contain almost the same amount of AgNPs, and suggests that zoledronate does not interfere with silver release.

\subsection{Cytotoxicity tests}

Cytotoxicity of the different synthesized materials were tested on MG63 cultured in direct contact with the samples. MG63 is a well known cell line of osteoblast derived from osteosarcoma, which are widely used in in vitro studies for biocompatibility and bioactivity, as they are well characterized and reproducible, and allow to limit as much as possible the variables in the in vitro model.

WST1 assay results at $72 \mathrm{~h}$ of culture are reported in Figure 4. According to ISO109935 , a reduction of cell viability greater than $30 \%$ compared to control indicates cytotoxicity of tested material. The viability of cells grown in direct contact with HA-5, HA-20, ZOL7-5, ZOL7-20, and ZOL14-5 samples showed no statistical difference (82\%, 72\%, $84 \%, 74 \%$, and $75 \%$ respectively) when compared to CTR- (viability considered as $100 \%)$. The other groups displayed a reduction of viability greater than $30 \%$. In particular HA-50, ZOL7-50, ZOL14-20, ZOL14-50 groups $(63 \%, 64 \%, 66 \%$, and $55 \%$ respectively) exhibited significant lower values of cell viability in comparison with CTR- AgNPs exerted some toxic effects: a slight difference was found between -5 and -20 samples $(\mathrm{p}<0.05)$, while a highly significant difference was detected when -50 was 
compared to both -5 and $-20(\mathrm{p}<0.0005)$. However, the comparison of the results of cell viability with the values of AgNPs content of the different samples (Table 1) allows to appreciate a role of zoledronate in reducing the cytotoxic effect of Ag. In fact, the amount of AgNPs sufficient to provoke a significant decrease of viability was lower in HA samples (1.1 wt\% in HA-50) than in bisphosphonate containing samples $(1.8,1.7$ and 2.4 wt\% in ZOL7-50, ZOL14-20 and ZOL14-50 respectively).

These results are supported by the evaluation of LDH released in culture medium, which is a measure of damaged membranes. No significant LDH release was detected in supernatants of HA-5, HA-20, ZOL7-5, ZOL7-20 and ZOL14-5 groups in comparison to CTR- after $72 \mathrm{~h}$ of culture (Figure 4).

Conversely, LDH values found in supernatants of HA-50, ZOL7-50, ZOL14-20, and ZOL14-50 groups were significantly higher than CTR-, confirming the negative effect of AgNPs at the highest concentration. In fact, the comparison of AgNPs containing groups shows that AgNPs in -50 samples significantly affected osteoblast culture in comparison to -5 and -20 samples ( $\mathrm{p}<0.0005)$ (both for HA and ZOL samples). Statistical analysis demonstrated that results of LDH were consistent and inversely correlated to WST1 proliferation assay (Pearson correlation coefficient $-0.727, \mathrm{p}<0.0005$ ). Figure S5 shows the images of Neutral Red staining of cells plated around all samples. The images confirm the data obtained by WST1 and LDH assays. In fact, osteoblasts on HA-5, HA20, ZOL7-5, ZOL7-20, and ZOL14-5, as well as on CTR-, grow as a monolayer, with numerous, well stained and regularly shaped cells. The images of osteoblast on HA-50, ZOL7-50, and ZOL14-20 show a decrease of cell number and a variable percentage of round shaped cells, even if the uptake of staining is remarkable. The majority of cells on 
ZOL14-50 did not uptake the color; they show important sign of lysis, and evident membrane damage.

The low molecular weight PEI used to synthesize AgNPs should not give any cytotoxicity [37]. However, in order to exclude any possible influence of PEI on cell response, cytotoxicity tests were performed on several samples, which were synthesized following the same procedure as that utilized for HA-20, HA-50, ZOL14-5 and ZOL14-50 but in absence of silver. These samples were labeled HA-20P, HA-50P, ZOL14-5P and ZOL1450P. All the samples showed no significantly different viability with respect to CTR-. In particular, at variance with the corresponding samples containing AgNPs, similar WST1 values were determined for HA-20P and HA-50P, and for ZOL14-5P and ZOL14-50P (Figure S6). Accordingly, LDH release from all the groups was not significantly different from CTR- (Figure S6), confirming that PEI presence does not damage the cells.

\subsection{Antibacterial susceptibility testing against bacterial strains}

In a preliminary set of experiments, the composite materials HA-5, HA-20, HAZOL7-5, HAZOL7-20, HAZOL14-5, and HAZOL14-20 were assayed for their antibacterial properties against reference ATCC strains including Gram positive ( $S$. aureus, $S$. epidermidis, E. faecalis) and Gram negative bacteria (E. coli, K. pneumoniae and P. aeruginosa). The antibacterial effect was determined by a standardized Kirby-Bauer disk diffusion method in which the inhibitory activity of the sample is proportional to the diameter of the bacterial-free zone around the disk-shaped compound. Results are reported in Table 2.

HA-AgNPs showed significant antibacterial activity against Gram positive bacteria with comparable bacterial-free zone diameters among the three species, and the inhibition slightly increased with AgNPs amount loaded on HA. On the other hand, among Gram 
negative bacteria the inhibitory effect was diversified for the different tested strains with a remarkable activity against $P$. aeruginosa, similar to that measured for the gentamicin positive control. All HAZOL-AgNPs samples displayed antibacterial properties against Gram positive bacteria comparable to those obtained for the HA-AgNPs while among Gram negative bacteria they showed a minimal activity towards $K$. pneumoniae but a high inhibitory activity against $P$. aeruginosa.

In the attempt to explain the different extent of activity of the composite materials on the selected reference panel, $\mathrm{MIC}_{90}$ values were determined for $\mathrm{Ag}^{+}$ions by using the broth microdilution methodology. As recommended by CLSI and EUCAST committees, the antimicrobial susceptibility assays were carried out in $\mathrm{MH}$ broth for all reference strains with the exception of E. faecalis; indeed, it was tested in BHI broth, following the ATCC instructions, as $\mathrm{MH}$ medium is not suitable for its cultivation. Once again a slightly different sensitivity to silver was displayed among the 5 bacterial strains tested in $\mathrm{MH}$ broth, with $\mathrm{MIC}_{90}$ values ranging from $1.5 \mu \mathrm{g} / \mathrm{mL}$ to $8.4 \mu \mathrm{g} / \mathrm{mL}$ for $S$. epidermidis and $K$. pneumoniae, respectively (Figure S7). $\mathrm{MIC}_{90}$ value for E. faecalis $(39.6 \mu \mathrm{g} / \mathrm{mL}$ ) cannot be directly compared with these values as the strain was cultured in a different medium and it has been previously stated that the susceptibility of isolates to $\mathrm{Ag}^{+}$varies considerably depending on media components as $\mathrm{NaCl}$ and proteins [45].

Notably, S. epidermidis and P. aeruginosa resulted the most susceptible strains among the reference bacteria in accordance to data obtained for the multifunctionalized materials in the disk diffusion assay. Of clinical relevance, S. epidermidis has recently emerged as opportunistic pathogens isolated from infected joints and its prevalence has increased mainly in infections associated to knee and hip arthroprostheses, revealing that this coagulase-negative Staphylococcus is progressively prevailing on S. aureus. Likewise, $P$. 
aeruginosa is the most prevalent Gram negative species isolated from infections associated to medical devices [46] and the treatment of these infections is particularly challenging because Gram negative bacteria possess a protective outer membrane that have an impact on their sensitivity to many different types of antibiotics. Moreover, it is well known that bacteria involved in osteoarticular chronic infections can live in a stationary phase, either intracellularly or within biofilms around the orthopaedic device. Inside the complex glycoproteic matrix of the biofilm, the low concentration of oxygen and nutrients leads to heterogeneous phenotypic changes in bacteria. In turn, this results in different antimicrobial tolerances to different families of antibiotics [47] In this context, we tested the antibacterial activity of the composite materials towards clinical isolates of S. epidermidis and $P$. aeruginosa obtained from chronic bone and prosthetic joint infections and presenting different antibiotic susceptibilities.

The selection of the samples for these tests, as well as for the co-culture in vitro tests reported in section 3.4, was based on the following requirements: the samples should not exhibit any cytotoxicity, should contain similar amounts of $\mathrm{Ag}$, whereas they should differ for the presence of zoledronate. On this basis, the tests were carried out on HA-20 (Ag content: $0.8 \mathrm{wt} \%$ ) and ZOL14-5 (Ag content: $0.9 \mathrm{wt} \%$, ZOL content: $8.6 \mathrm{wt} \%$ ), which did not exhibit cytotoxicity (Figure 4). The antibiotic-resistance profile of each clinical strain and the antibacterial activities of the composite materials are reported in Table 3. Both HA-AgNPs (HA-20) and HAZOL-AgNPs (HAZOL14-5) revealed a broadspectrum antimicrobial activity against all the tested clinical isolates, regardless of their antibiotic-resistance profiles. Indeed, the composite materials displayed inhibitory properties towards the two MSSE strains, and, most importantly, towards the three MRSE strains that were resistant to erythromycin or gentamicin or levofloxacin or 
trimethoprim/sulfamethoxazole, in addition to oxacillin and all other $\beta$-lactam and cephalosporin antibiotics that share structural similarity with methicillin. Furthermore, the composite materials inhibited bacterial growth of all the isolates of $P$. aeruginosa including the two presenting a MDR phenotype.

An important consideration with regard to the use of $\mathrm{Ag}$ as additive to many medical devices is the potential for the development of bacterial resistance. Molecular basis for $\mathrm{Ag}^{+}$resistance have been characterized and include determinants located on plasmids and bacterial chromosome encoding periplasmatic efflux transporter and periplasmatic multimetal-binding proteins [48]. To date, failure to select $\mathrm{Ag}^{+}$resistant mutants in vitro upon prolonged metal exposure [45], and the little to no evidence of significant phenotypic $\mathrm{Ag}^{+}$-resistant expression in clinical isolates [49] suggest that silver-based materials remain a viable option for the prevention and treatment of pathogens including MDR bacteria [19]. There would be anyhow benefit in surveillance programs to monitor the emergence and spread of silver-resistant strains.

\subsection{Co-cultures}

In the present study $\mathrm{OB}$ and $\mathrm{OC}$ derived from monocytes of osteoporotic subjects were co-cultured up to 14 days, in direct contact with tested materials, to evaluate the effects of local administration of HA functionalized with ZOL and AgNPs. In order to evaluate the single and coupled effects of AgNPs and ZOL, the tests were carried out on HA, HA20, ZOL14, ZOL14-5.

WST1 data demonstrated that ZOL (Figure 5), at the tested concentration, did not exhibit cytotoxic effect on $\mathrm{OB}$, which showed regular proliferation and did not differ from HA, as reference material, and CTR group. In fact OB cultured on ZOL14ZOL14-5 had a viability of $95 \%$ and $88 \%$ respectively, compared to $\mathrm{CTR}=100 \%$. On the other hand, the 
addition of AgNPs to pure HA (HA-20) had different results with respect to those obtained at three days in monoculture: OB growth in co-culture at 14 days was inhibited and the value of viability (64\%) was significantly lower in comparison with other groups. The reduced cell viability on HA-20 can be ascribed to the presence of AgNPs, which have been reported to induce cytotoxic effects by increasing NO-derived reactive species, apoptosis and cell death [50]. On the other hand, the higher value of OB viability measured on ZOL14-5 (which contains about the same amount of AgNPs as HA-20, and displays similar Ag release) suggests that zoledronate hinders the cytotoxic effect of AgNPs. However, OB appeared attached and well spread, and rich of filopodia on all the samples, as shown in the SEM images reported in Figure S8 for HA-20 and ZOL14-5. Osteoclastogenesis from osteoclast precursors was widely affected by both ZOL and AgNPs, as shown in Figure 5. OC grown in presence of HA-20, ZOL14 and ZOL14-5 showed significant very lower viability when compared to OC cultured with HA and CTR. Moreover TRAP staining of monocytes revealed that the number of multinucleated cells in samples contaning AgNPs and/or ZOL (HA-20: 26\%, ZOL14: 8\%, ZOL14-5: $6 \%$ ) is remarkably reduced in comparison with HA (98\%) and CTR (assumed as 100\%). Figure 6 shows the results of OB metabolic activity. ALP and OSTC represent markers of OB differentiation. ALP activity increases in the early stage of differentiation, while OSTC is involved in the late phase of mineralization. ALP does not seem to be significantly affected by ZOL and AgNPs, when compared to CTR. Conversely, OSTC levels in ZOL14 and ZOL14-5 were significantly higher than in the other groups. The comparison of the data obtained for HA-20 and ZOL14-5 respectively with those of HA and ZOL14 demonstrates that AgNPs presence does not modify the production of OSTC. ZOL14 showed a significantly higher level of OPG and ZOL14-5 a significant lower 
RANKL, with respect to the samples without ZOL. As a result, the OPG/RANKL ratio was higher on both ZOL14 and ZOL14-5 than on the other samples, confirming that ZOL is an effective inhibitor of osteoclastogenesis through the OPG/RANKL pathway. Results of OPG/RANKL ratio inversely correlated with OC viability $(-0.766, \mathrm{p}<0.0005)$ and differentiation (TRAP \%: 0.704, $\mathrm{p}<0.005$ ).

IL-6 and TNF- $\alpha$ activity were also investigated as they play an important role in promoting inflammation and are related to bone resorption activity in osteoporosis. AgNPs seems to stimulate both factors. In agreement with viability results (Figure 5), HA-20 significantly enhanced the production of IL-6 and TNF- $\alpha$, with respect to other groups. However, the co-presence of ZOL reduced this effect. In fact, IL-6 and TNF- $\alpha$ levels were significantly lower in ZOL14 and ZOL14-5 when compared to HA-20, whereas no differences were found for TNF- $\alpha$ among ZOL groups and CTR (Figure 7). The in vitro model of $\mathrm{OB}$ and $\mathrm{OC}$ co-culture allows to study the interplay of bone cells when interfaced with biomaterials, and to understand the effects of different molecules on bone turnover. According to our data, it could be hypothesized that the low number of both $\mathrm{OB}$ and $\mathrm{OC}$ in HA-20 is related to cell death or damage by the cytotoxic effect of AgNPs that also stimulated and activated proinflammatory molecules as IL-6 and TNF$\alpha$. However, OB activity parameters, normalized for cell viability (WST1), did not show significant differences between HA and HA-20, suggesting that the surviving fraction of OB were not suffering because of AgNPs presence. It is worth mentioning that, in agreement with cytotoxicity data, the presence of zoledronate hinders the effect of AgNPs on viability, IL-6 and TNF- $\alpha$ (Figures 6,7), without affecting its antimicrobial activity (Table 3).

This item was downloaded from IRIS Università di Bologna (https://cris.unibo.it/) 
The influence of the bisphosphonate in ZOL14 and ZOL14-5 groups on bone metabolism is evident both as direct action on $\mathrm{OC}$ viability and as indirect influence on OC differentiation. In fact, the improvement of OB differentiation and activity is testified not only by the high level of OSTC, but also by the increase of OPG/RANKL ratio, that contribute to downregulate OC proliferation and differentiation. No differences of activity were found between ZOL14 and ZOL14-5, demonstrating that the utilized content of AgNPs as anti-bacterial agent does not interfere with the positive influence of the bisphosphonate.

\section{Conclusions}

Results of the present study show that AgNPs can be loaded onto zoledronate functionalized HA engendering composite materials with multiple biological properties. HAZOL promotes in vitro osteoblast viability and activity and inhibits osteoclast proliferation and differentiation; silver nanoparticles inhibit the bacterial growth of multidrug resistant Gram positive and negative bacteria in vitro. Combination of these bioactivities may represent a pioneering strategy in the treatment of implant-associated infections, in particular in selected categories of patients, as adults with cystic fibrosis (CF). Indeed, the survival gains achieved in CF patients have led to the emergence of delayed complications, one of each is bone disease characterized by low mineral density [51], usually treated with oral or intravenous bisphosphonate preparations. Increased bone resorption and disturbances in bone formation are most frequent during infectious episodes [52]. HAZOL-AgNPs, integrating the positive effects of BPs on bone metabolism and the antimicrobial prophylactic activity of AgNPs on the development of 
bone-associated infections, can represent a new tool to counteract implant-associated infections.

\section{Acknowledgements}

This research was carried out with the financial support of the University of Bologna (FARB 2012) and of Rizzoli Orthopaedic Institute (5 X 1000 year 2015 n. 6879). The authors gratefully thank Dr. Stefano Cerini, Dr. Alberto Mucchi and Flavien Garcia Moran for experimental support. 


\section{References}

[1] E. Verron, J.M. Bouler, J. Guicheux, Controlling the biological function of calcium phosphate bone substitutes with drugs, Acta Biomater. 8 (2012) 3541-3551.

[2] A. Bigi, E. Boanini, M. Gazzano, Ion Substitution in Biological and Synthetic Apatites

- in Biomineralization and Biomaterials, Foundamentals and applications, C. Aparicio, M.P. Ginebra (Eds.), Woodhead Publishing, Sawston, Cambridge, UK, 2015, pp 235266.

[3] F. Paladini, M. Pollini, A. Sannino, L. Ambrosio, Metal-based antibacterial substrates for biomedical applications, Biomacromolecules 16 (2015) 1873-1885.

[4] E. Boanini, P. Torricelli, F. Sima, E. Axente, M. Fini, I.N. Mihailescu, A. Bigi, Strontium and zoledronate hydroxyapatites graded composite coatings for bone prostheses, J. Colloid Interface Sci. 448 (2015) 1-7.

[5] E. Boanini, P. Torricelli, M. Gazzano, M. Fini, A. Bigi, The effect of zoledronatehydroxyapatite nanocomposites on osteoclasts and osteoblast-like cells in vitro, Biomaterials 33 (2012) 722-730.

[6] E. Boanini, P. Torricelli, M. Gazzano, M. Fini, A. Bigi, Crystalline calcium alendronate obtained by octacalcium phosphate digestion: a new chance for local treatment of bone loss diseases? Adv. Mater. 25 (2013) 4605-4611.

[7] R.G.G. Russell, Bisphosphonates: the First 40 Years, Bone 49 (2011) 2-19.

[8] V. Stresing, F. Daubiné, I. Benzaid, H. Mönkkönen, P. Clézardin, Bisphosphonates in cancer therapy, Cancer Lett. 257 (2007) 16-35.

This item was downloaded from IRIS Università di Bologna (https://cris.unibo.it/) 
[9] G.H. Nancollas, R. Tang, R.J. Phipps, Z. Henneman, S. Gulde, W. Wu, A. Mangood, R.G. Russell, F.H. Ebetino, Novel insights into actions of bisphosphonates on bone: differences in interactions with hydroxyapatite, Bone 38 (2006) 617-627.

[10] M.J. Rogers, From molds and macrophages to mevalonate: a decade of progress in understanding the molecular mode of action of bisphosphonates, Calcif. Tissue Int. 75 (2004) 451-461.

[11] G. Favia, G.P. Pilolli, E. Maiorano, Histologic and histomorphometric features of bisphosphonate-related osteonecrosis of the jaws: an analysis of 31 cases with confocal laser scanning microscopy, Bone 45 (2009) 406-413.

[12] R. Rizzoli, K. Åkesson, M. Bouxsein, J.A. Kanis, N. Napoli, S. Papapoulos, J.Y. Reginster, C. Cooper, Subtrochanteric fractures after long-term treatment with bisphosphonates: a european society on clinical and economic aspects of osteoporosis and osteoarthritis, and international osteoporosis foundation working group report, Osteoporos. Int. 22 (2011) 373-390.

[13] E. Verron, J.M. Bouler, Is bisphosphonate therapy compromised by the emergence of adverse bone disorders? Drug Discov. Today 19 (2014) 312-319.

[14] L. Kyllönen, M. D’Este, M. Alini, D. Eglin, Local drug delivery for enhancing fracture healing in osteoporotic bone, Acta Biomater. 11 (2015) 412-434.

[15] C. Faucheux, E. Verron, A. Soueidan, S. Josse, M.D. Arshad, P. Janvier, P. Pilet,; J.M. Bouler, B. Bujoli, J. Guicheux, Controlled release of bisphosphonate from a calcium phosphate biomaterial inhibits osteoclastic resorption in vitro, J. Biomed. Mater. Res. A, 89A (2009) 46-56.

This item was downloaded from IRIS Università di Bologna (https://cris.unibo.it/) 
[16] E. Boanini, P. Torricelli, M. Gazzano, E. Della Bella, M. Fini, A. Bigi, Combined effect of strontium and zoledronate on hydroxyapatite structure and bone cell responses, Biomaterials 35 (2014) 5619-5626.

[17] W. Toy, L. Macera, Evidence-based review of silver dressing use on chronic wounds, J. Am. Acad. Nurse Pract. 23 (2011) 183-192.

[18] Y. Teow, P.V. Asharani, M.P. Hande, S. Valiyaveettil, Health impact and safety of engineered nanomaterials, Chem. Commun. 47 (2011) 7025-7038.

[19] M.K. Rai, S.D. Deshmukh, A.P. Ingle, A.K.J. Gade, Silver nanoparticles: the powerful nanoweapon against multidrug-resistant bacteria, Appl. Microbiol. 112 (2012) $841-852$.

[20] S. Bose, S. Tarafder, Calcium phosphate ceramic systems in growth factor and drug delivery for bone tissue engineering: a review, Acta Biomater. 8 (2012) 1401-1421.

[21] S. Shrivastava, T. Bera, A. Roy, G. Singh, P. Ramachandrarao, D. Dash, Characterization of enhanced antibacterial effects of novel silver nanoparticles, Nanotechnology 18 (2007) 225103.

[22] A.K. Suresh, D.A. Pelletier, W. Wang, J.L. Morrell-Falvey, B. Gu, M.J. Doktycz, Cytotoxicity induced by engineered silver nanocrystallites is dependent on surface coatings and cell types, Langmuir 28 (2012) 2727-2735.

[23] Q.L. Feng, J. Wu, G.Q. Chen, F.Z. Cui, T.N. Kim, J. O. Kim, A mechanistic study of the antibacterial effect of silver ions on Escherichia coli and Staphylococcus aureus, J. Biomed. Mater. Res. 52 (2000) 662-668.

[24] S. Sivolella, E. Stellini, G. Brunello, C. Gardin, L. Ferroni, E. Bressan, B. Zavan, Silver nanoparticles in alveolar bone surgery devices, J. Nanomater. (2012), 975842.

This item was downloaded from IRIS Università di Bologna (https://cris.unibo.it/) 
[25] M.V. Park, A.M. Neigh, J.P. Vermeulen, L.J. de la Fonteyne, H.W. Verharen, J.J. Briedé, H. van Loveren, W. H. de Jong, The effect of particle size on the cytotoxicity, inflammation, developmental toxicity and genotoxicity of silver nanoparticles, Biomaterials 32 (2011) 9810-9817.

[26] J. Gallo, M. Holinka, C.S. Moucha, Antibacterial Surface Treatment for Orthopaedic Implants, Int. J. Mol. Sci. 15 (2014) 13849-13880.

[27] S.A. Brennan, C. Ní Fhoghlú, B.M. Devitt, F.J. O’Mahony, D. Brabazon, A. Walsh, Silver nanoparticles and their orthopaedic applications, Bone Joint J. 97-B (2015) 582589.

[28] G.A. Fielding, M. Roy, A. Bandyopadhyay, S. Bose, Antibacterial and biological characteristics of silver containing and strontium doped plasma sprayed hydroxyapatite coatings, Acta Biomater. 8 (2012) 3144-3152.

[29] W. Chen, S. Oh, A.P. Ong, N. Oh, Y. Liu, H.S. Courtney, M. Appleford, J.L. Ong, Antibacterial and osteogenic properties of silver-containing hydroxyapatite coatings produced using a sol gel process, J. Biomed. Mater. Res. A 82A (2007) 899-906.

[30] P.N. Lim, L. Chang, B.Y. Tay, V. Guneta, C. Choong, B. Ho, E.S. Thian, Proposed mechanism of antibacterial action of chemically modified apatite for reduced bone infection, ACS Appl. Mater. Interfaces 6 (2014) 17082-17092.

[31] M. Furkó, K. Balázsi, C. Balázsi, Comparative study on preparation and characterization of bioactive coatings for biomedical applications - a review on recent patents and literature, Rev. Adv. Mater. Sci. 48 (2017) 25-51.

[32] S.K. Arumugam, T.P. Sastry, B. Sreedhar, A.B. Mandal, One step synthesis of silver nanorods by autoreduction of aqueous silver ions with hydroxyapatite: an 
inorganic-inorganic hybrid nanocomposite, J. Biomed. Mater. Res. A 80A (2007) 391398.

[33] M. Diaz, F. Barba, M. Miranda, F. Guitian, R. Torrecillas, J.S. Moya, Synthesis and antimicrobial activity of a silver-hydroxyapatite nanocomposite, J. Nanomater. 2009, Article ID 498505, 6 pages.

[34] M. Li, X. Liu, Z. Xu, K.W.K. Yeung, S. Wu, Dopamine modified organic-inorganic hybrid coating for antimicrobial and osteogenesis, ACS Appl. Mater. Interfaces 8 (2016) $33972-33981$.

[35] L. Yan, Y. Xiang, J. Yu, Y. Wang, W. Cui, Fabrication of antibacterial and antiwear hydroxyapatite coatings via in situ chitosan-mediated pulse electrochemical deposition, ACS Appl. Mater. Interfaces 9 (2017) 5023-5030

[36] E. Boanini, P. Torricelli, M.C. Cassani, G.A. Gentilomi, B. Ballarin, K. Rubini, F. Bonvicini, A. Bigi, Cationic-anionic polyelectrolyte interaction as a tool to graft silver nanoparticles on hydroxyapatite crystals and prevent cytotoxicity, RSC Adv. 4 (2014) $645-652$

[37] M. Thomas, Q. Ge, J.J. Lu, J. Chen, A.M. Klibanov, Cross-linked small polyethylenimines: while still nontoxic, deliver dna efficiently to mammalian cells in vitro and in vivo, Pharmaceutical Research 22 (2005) 373-380.

[38] M. Jevon, T. Hirayama, M.A. Brown, J.A. Wass, A. Sabokbar, S. Ostelere, N.A.Athenasou, Osteoclast formation from circulating precursors in osteoporosis, Scand. J. Rheumatol. 32 (2003) 95-100.

[39] F. Salamanna, M. Maglio, G. Giavaresi, S. Pagani, R. Giardino, M. Fini, In vitro method for the screening and monitoring of estrogen-deficiency osteoporosis by targeting peripheral circulating monocytes, Age 37 (2015) 9819.

This item was downloaded from IRIS Università di Bologna (https://cris.unibo.it/)

When citing, please refer to the published version. 
[40] R.F. Jiménez-Ortega E.G. Ramírez-Salazar, A.Y. Parra-Torres, S.A. MuñozMontero, C. Rangel-Escareňo, I. Salido-Guadarrama, M. Rodriguez-Dorantes, M. Quiterio, J. Salmerón, R. Velázquez-Cruz, Identification of microRNAs in human circulating monocytes of postmenopausal osteoporotic Mexican-Mestizo women: A pilot study. Exp Ther Med. 14 (2017) 5464-5472.

[41] J. Kuljanin, I. Janković, J. Nedeljković, D. Prstojević, V. Marinković, Spectrophotometric determination of alendronate in pharmaceutical formulations via complex formation with Fe(III) ions, J. Pharm. Biomed. Anal. 28 (2002) 1215-1220.

[42] A. Croxatto, G. Prod'hom, G. Greub, Applications of MALDI-TOF mass spectrometry in clinical diagnostic microbiology, FEMS Microbiol. Rev. 36 (2012) 380407.

[43] EUCAST: The European Committee on Antimicrobial Susceptibility Testing. Breakpoint tables for interpretation of MICs and zone diameters. Version 6.0, 2016. http://www.eucast.org.

[44] Clinical and Laboratory Standards Institute. Performance Standards for Antimicrobial Susceptibility Testing; Twenty-fifth Informational Supplement. CLSI document M100-S25 (2015)

[45] C.P. Randall, L.B. Oyama, J.M. Bostock, I. Chopra, A.J. O’Neill, The silver cation $\left(\mathrm{Ag}^{+}\right)$: antistaphylococcal activity, mode of action and resistance studies, J. Antimicrob. Chemother. 68 (2013) 131-138.

[46] L. Montanaro, P. Speziale, D. Campoccia, S. Ravaioli, I. Cangini, G. Pietrocola, S. Giannini, C.R. Arciola, Scenery of Staphylococcus implant infections in orthopedics, Future Microbiol. 6 (2011) 1329-1349.

This item was downloaded from IRIS Università di Bologna (https://cris.unibo.it/)

When citing, please refer to the published version. 
[47] A. Ribera, E. Benavent, J. Lora-Tamayo, F. Tubau, S. Pedrero, X. Cabo, J. Ariza, O. Murillo, Osteoarticular infection caused by MDR Pseudomonas aeruginosa: the benefits of combination therapy with colistin plus $\beta$-lactams, J. Antimicrob. Chemother. 70 (2015) $3357-3365$.

[48] S. Silver, Bacterial silver resistance: molecular biology and uses and misuses of silver compounds, FEMS Microbiol. Rev. 27 (2003) 341-353.

[49] P.J. Finley, R. Norton, C. Austin, A. Mitchell, S. Zank, P. Durham, Unprecedented silver resistance in clinically isolated Enterobacteriaceae: major implications for burn and wound management, Antimicrob. Agents Chemother. 59 (2015) 4734-4741

[50] E. Zielinska, C. Tukaj, M.W. Radomski, I. Inkielewicz-Stepniak, Molecular mechanism of silver nanoparticles-induced human osteoblast cell death: protective effect of inducible nitric oxide synthase inhibitor, PLoS One 11 (2016) e0164137.

[51] R.M. Javier, J. Jaquot, Bone disease in cystic fibrosis: what's new? Joint Bone Spine 78 (2011) 445-450.

[52] M. Marquette, C.S. Haworth, Bone health and disease in cystic fibrosis, Paediatr. Respir. Rev. 20S (2016) 2-5. 


\section{Captions to the Figures.}

Figure 1. XRD patterns of the different samples obtained through interaction of the apatitic materials with $50 \mathrm{~mL}$ of AgNPs suspension. The most intense peak of $\mathrm{Ag}$ is indicated with $\left(^{*}\right)$; all the other peaks are characteristic of HA.

Figure 2. TEM images of the different samples obtained through interaction of the apatitic materials with $50 \mathrm{~mL}$ of AgNPs suspension. Scale bars are $200 \mathrm{~nm}$ in all images for direct comparison.

Figure 3. Ag cumulative release from HA-20 and ZOL14-5 as a function of time.

Figure 4. WST1 assay and LDH release of MG63 72 hours of culture on experimental samples and CTRs (negative CTR-, DMEM only; positive CTR+, DMEM+0.05\% phenol solution). Values are reported as mean $\pm \mathrm{SD}(* \mathrm{p}<0.05 ; * * \mathrm{p}<0.005 ; * * * \mathrm{p}<0.0005)$. WST1. HA-50, ZOL7-50, ZOL14-20, ZOL14-50 vs CTR- $(\mathrm{p}<0.05)$; CTR+ vs CTR$(\mathrm{p}<0,0005)$.

LDH. ZOL7-50 vs CTR- (p<0,05); HA-50, ZOL14-20 vs CTR- $(\mathrm{p}<0.005)$, ZOL14-50, CTR+ vs CTR- $(\mathrm{p}<0,0005)$.

Figure 5. Effect of ZOL14 and AgNPs on viability of OB, and OC by WST1 test after 14 days of co-culture compared to HA as reference material and to CTR group.

The Tamhane post hoc multiple comparison test was performed to detect significant differences among groups $(* \mathrm{p}<0.05 ; * * \mathrm{p}<0.005 ; * * * \mathrm{p}<0.0001)$.

OB: *HA-20 vs HA; ***HA-20 vs ZOL14, CTR;

OC: ***HA-20 vs HA, ZOL14, ZOL14-5, CTR; *ZOL14 vs ZOL14-5; ***ZOL14, ZOL14-5 vs HA, CTR.

This item was downloaded from IRIS Università di Bologna (https://cris.unibo.it/)

When citing, please refer to the published version. 
Figure 6. Metabolic activity of co-cultured cells measured in culture supernatant after 14 days of co-culture with HA, HA-20, ZOL14, ZOL14-5, and CTR. The Tamhane post hoc multiple comparison test was performed to detect significant differences among groups $(* \mathrm{p}<0.05 ; * * \mathrm{p}<0.005 ; * * * \mathrm{p}<0.0001)$.

ALP: not significant

OSTC: *ZOL14 vs HA; **ZOL14 vs HA-20, CTR; ***ZOL14-5 vs HA, HA-20, ZOL14; OPG: *ZOL14 vs HA, HA-20, CTR;

RANKL: *ZOL14-5 vs HA, HA-20; **ZOL-14-5 vs CTR;

OPG/RANKL ratio: ***ZOL14 vs HA, HA-20, CTR; *ZOL14-5 vs HA-20, CTR

Figure 7. Evaluation of pro-inflammatory cytokine IL-6 and growth factor TNF- $\alpha$ in OB and OC co-cultured with HA, HA-20, ZOL14, ZOL14-5, and CTR after 14 days of culture. The Tamhane post hoc multiple comparison test was performed to detect significant differences among groups $(* \mathrm{p}<0.05 ; * * \mathrm{p}<0.005 ; * * * \mathrm{p}<0.0001)$. IL-6: *HA vs HA-20; ***ZoL14 vs HA-20, CTR; **ZOL-14-5 vs HA-20, CTR; TNF- $\alpha$ : *HA-20 vs HA; **HA-20 vs ZOL14, ZOL14-5, CTR. 